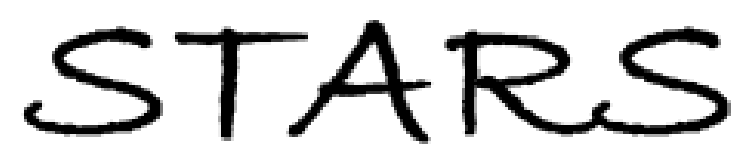

University of Central Florida

STARS

$1-1-2011$

\title{
Anderson localization in optical waveguide arrays with off- diagonal coupling disorder
}

\author{
Lane Martin \\ University of Central Florida \\ Giovanni Di Giuseppe \\ University of Central Florida \\ Armando Perez-Leija \\ University of Central Florida \\ Robert Keil
}

Felix Dreisow

Find similar works at: https://stars.library.ucf.edu/facultybib2010

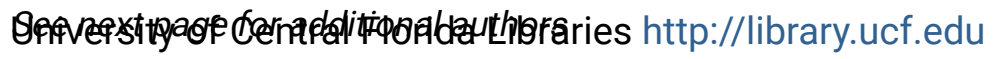

This Article is brought to you for free and open access by the Faculty Bibliography at STARS. It has been accepted for inclusion in Faculty Bibliography 2010 s by an authorized administrator of STARS. For more information, please contact STARS@ucf.edu.

\section{Recommended Citation}

Martin, Lane; Di Giuseppe, Giovanni; Perez-Leija, Armando; Keil, Robert; Dreisow, Felix; Heinrich, Matthias; Nolte, Stefan; Szameit, Alexander; Abouraddy, Ayman F.; Christodoulides, Demetrios N.; and Saleh, Bahaa E. A., "Anderson localization in optical waveguide arrays with off-diagonal coupling disorder" (2011).

Faculty Bibliography 2010s. 1633.

https://stars.library.ucf.edu/facultybib2010/1633

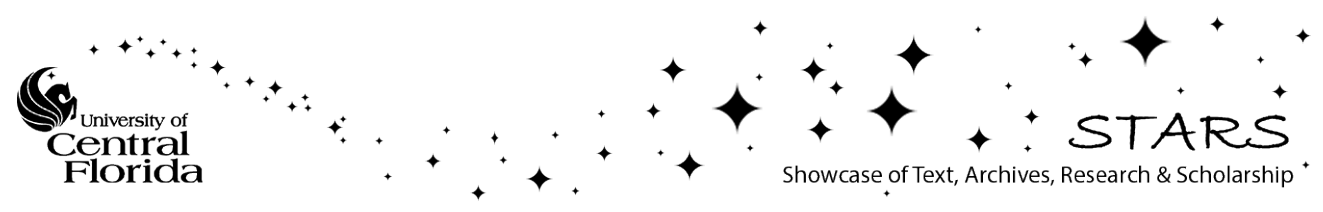




\section{Authors}

Lane Martin, Giovanni Di Giuseppe, Armando Perez-Leija, Robert Keil, Felix Dreisow, Matthias Heinrich, Stefan Nolte, Alexander Szameit, Ayman F. Abouraddy, Demetrios N. Christodoulides, and Bahaa E. A. Saleh 


\title{
Anderson localization in optical waveguide arrays with off-diagonal coupling disorder
}

\author{
Lane Martin, ${ }^{1}$ Giovanni Di Giuseppe, ${ }^{1,2}$ Armando Perez-Leija, ${ }^{1,3}$ \\ Robert Keil, ${ }^{4}$ Felix Dreisow, ${ }^{4}$ Matthias Heinrich, ${ }^{4}$ Stefan Nolte, ${ }^{4}$ \\ Alexander Szameit, ${ }^{5}$ Ayman F. Abouraddy, \\ Demetrios N. Christodoulides, ${ }^{1}$ and Bahaa E. A. Saleh ${ }^{1, *}$ \\ ${ }^{1}$ CREOL, The College of Optics \& Photonics, University of Central Florida, Orlando, FL \\ 32816, USA \\ ${ }^{2}$ School of Science and Technology, Physics Division, University of Camerino, 62032 \\ Camerino (MC), Italy \\ ${ }^{3}$ INAOE, Coordinacion de Optica, A.P. 51 y 216, 72000 Puebla, Puebla, Mexico \\ ${ }^{4}$ Institute of Applied Physics, Friedrich-Schiller-Universität Jena, Max-Wien-Platz 1, 07743 \\ Jena, Germany \\ ${ }^{5}$ Solid State Institute and Physics Department, Technion, 32000 Haifa, Israel \\ *besaleh@creol.ucf.edu
}

\begin{abstract}
We report on the observation of Anderson wave localization in one-dimensional waveguide arrays with off-diagonal disorder. The waveguide elements are inscribed in silica glass, and a uniform random distribution of coupling parameters is achieved by a precise variation of the relative waveguide positions. In the absence of disorder we observe ballistic transport as expected from discrete diffraction in periodic arrays. When off-diagonal disorder is deliberately introduced into the array we observe Anderson localization. The strength of the localization signature increases with higher levels of disorder.
\end{abstract}

(C) 2011 Optical Society of America

OCIS codes: (070.7345) Wave propagation; (130.2790) Guided waves; (270.1670) Coherent optical effects.

\section{References and links}

1. P. W. Anderson, "Absence of diffusion in certain random lattices," Phys. Rev. 109, 1492-1505 (1958).

2. A. Lagendijk, B. van Tiggelen, and D. S. Wiersma, "Fifty years of Anderson localization," Phys. Today 62(8), 24-29 (2008)

3. C. M. Soukoulis and E. N. Economou, "Off-diagonal disorder in one-dimensional systems," Phys. Rev. B 24, 5698-5702 (1981).

4. P. Erdös and R. C. Herndon, "Theories of electrons in one-dimensional disordered systems," Adv. Phys. 31, 65-163 (1982)

5. D. N. Christodoulides, F. Lederer, and Y. Silberberg, "Discretizing light behaviour in linear and nonlinear waveguide lattices," Nature 424, 817-823 (2003).

6. R. Morandotti, U. Peschel, J. S. Aitchison, H. S. Eisenberg, and Y. Silberberg, "Experimental observation of linear and nonlinear optical Bloch oscillations," Phys. Rev. Lett. 100, 170506 (2008).

7. T. Pertsch, P. Dannberg, W. Elflein, A. Braeuer, and F. Lederer, "Optical Bloch oscillations in temperature tuned waveguide arrays," Phys. Rev. Lett. 83, 4752-4755 (1999).

8. H. Trompeter, W. Krolikowski, D. N. Neshev, A. S. Desyatnikov, A. A. Sukhorukov, Yu. S. Kivshar, T. Pertsch, U. Peschel, and F. Lederer, "Bloch oscillations and Zener tunneling in two-dimensional photonic lattices," Phys. Rev. Lett. 96, 053903 (2006).

\#145189 - \$15.00 USD Received 1 Apr 2011; revised 15 May 2011; accepted 26 May 2011; published 29 Jun 2011

(C) 2011 OSA

4 July 2011 / Vol. 19, No. 14 / OPTICS EXPRESS 13636 
9. H. B. Perets, Y. Lahini, F. Pozzi, M. Sorel, R. Morandotti, and Y. Silberberg, "Realization of quantum walks with negligible decoherence in waveguide lattices," Phys. Rev. Lett. 100, 170506 (2008).

10. S. Longhi, M. Marangoni, M. Lobino, R. Ramponi, P. Laporta, E. Cianci, and V. Foglietti, "Observation of dynamic localization in periodically curved waveguide arrays," Phys. Rev. Lett. 96, 243901 (2006).

11. K. Shandarova, C. E. Rüter, D. Kip, K. G. Makris, D. N. Christodoulides, O. Peleg, and M. Segev, "Experimental observation of Rabi oscillations in photonic lattices," Phys. Rev. Lett. 102, 123905 (2009).

12. A. Perez-Leija, H. Moya-Cessa, A. Szameit, and D. N. Christodoulides, "Glauber-Fock photonic lattices," Opt. Lett. 35, 2409-2411 (2010).

13. Y. Lahini, A. Avidan, F. Pozzi, M. Sorel, R. Morandotti, D. N. Christodoulides, and Y. Silberberg, "Anderson localization and nonlinearity in one-dimensional disordered photonic lattices," Phys. Rev. Lett. 100, 013906 (2008).

14. T. Schwartz, G. Bartal, S. Fishman, and M. Segev, "Transport and Anderson localization in disordered twodimensional photonic lattices," Nature 446, 52-55 (2007).

15. A. Szameit, Y. V. Kartashov, P. Zeil, F. Dreisow, M. Heinrich, R. Keil, S. Nolte, A. Tünnermann, V. A. Vysloukh, and L. Torner, "Wave localization at the boundary of disordered photonic lattices," Opt. Lett. 35, 1172-1174 (2010).

16. Y. Lahini, Y. Bromberg, Y. Shechtman, A. Szameit, D. N. Christodoulides, R. Morandotti and Y. Silberberg, "Hanbury Brown and Twiss correlations of Anderson localized waves," arXiv:1104.0592v1.

17. J. B. Pendry, "Off-diagonal disorder and 1D localization,” J. Phys. C: Solid State Phys. 15, 5773-5778 (1982).

18. D. Blömer, A. Szameit, F. Dreisow, T. Schreiber, S. Nolte, and A. Tünnermann, "Nonlinear refractive index of fs-laser-written waveguides in fused silica," Opt. Express 14, 2151-2157 (2006).

19. A. Szameit, F. Dreisow, T. Pertsch, S. Nolte, and A. Tünnermann, "Control of directional evanescent coupling in fs laser written waveguides," Opt. Express 15, 1579-1587 (2007).

20. F. M. Izrailev, T. Kottos, A. Politi, and G. P. Tsironis, "Evolution of wave packets in quasi-one-dimensional and one-dimensional random media: diffusion versus localization,” Phys. Rev. E 55, 4951-4963 (1997).

\section{Introduction}

Anderson localization is ubiquitous in wave physics. This process naturally arises in any random lattice system and is known to result from the interference between multiple scattering events. Under strong disorder conditions this interference can become so severe that it entirely holds the transport of a quantum mechanical wave-packet. In this regime, Anderson localization occurs. While in higher dimensions the transition from ballistic to Anderson localization is preceded by diffusion, in 1D-systems this effect can be directly induced even in the presence of weak disorder [1,2]. Over the years Anderson localization has been analyzed in the literature under both diagonal [1] and off-diagonal disorder conditions [3,4].

Lattices of coupled optical waveguides provide a versatile platform for manipulating the flow of light [5]. In recent years such arrays have been used to directly observe and study optical analogs of many fundamental quantum mechanical effects like Bloch oscillations [6,7], Zener tunneling [8], continuous-time quantum random walks [9], and other processes [10-12]. Another example is Anderson localization that has been directly observed for light propagating in one- (1D) and two-dimensional (2D) arrays of coupled waveguides [13,14]. These observations have been demonstrated for the case of diagonal disorder, i.e., the waveguide propagation constants are randomized (by randomizing the sizes of the waveguides), while keeping the coupling coefficients between adjacent waveguides approximately constant (by keeping the waveguide separations constant). Anderson localization for off-diagonal disordered waveguide arrays has been reported for the first time in Ref. [15]. In such an array, the waveguide elements are all identical (i.e. have the same propagation constant) while the coupling coefficients are varied by changing their relative positions. In Ref. [15], disorder-induced localization by averaging over many array samples having the same degree of disorder was observed in 1D photonic lattices. We report here the observation of Anderson localization in a 1D optical waveguide array with off-diagonal disorder having a uniform random distribution of coupling coefficients. We prove through experimental observation and calculation that the shift invariance of the statistical characteristics of the waveguide disorder allows one to replace statistical averaging over multiple sample realizations with shifting the input waveguide excited in the same sample real-

\#145189 - \$15.00 USD Received 1 Apr 2011; revised 15 May 2011; accepted 26 May 2011; published 29 Jun 2011

(C) 2011 OSA

4 July 2011 / Vol. 19, No. 14 / OPTICS EXPRESS 13637 
ization. Furthermore, we examine the effect of the waveguide array length on the propagation dynamics in both the periodic and the disordered arrays. We would like to emphasize that while both diagonal and off-diagonal disorder can lead to Anderson localization, there are still qualitative differences between them [16]. One such aspect manifests itself in the level of disorder needed to accomplish 1D localization. In general, for off-diagonal disorder, stronger level of randomness is necessary compared to that required for diagonal disorder if the same localization length is to be attained. Reference [17] highlights this issue among other distinguishing traits of off-diagonal disorder.

The waveguides used here are fabricated by use of intense infrared femtosecond laser pulses focused inside transparent silica $[18,19]$. The utilized glass allows us to use light of wavelengths shorter than those used in $\mathrm{AlGaAs}$ waveguides in previous demonstrations of Anderson localization of light in arrays with diagonal disorder. Glass waveguides also mitigate the low coupling efficiency of light into high-refractive-index $\left(n_{A l G a A s} \approx 3.3\right)$ waveguides. In our arrays, the waveguides are all identical, i.e. they all have the same propagation constant, while the coupling coefficients are randomized by changing the relative positions of the waveguides. We observe that extended states in a periodic waveguide array become exponentially localized states when the localization length is shorted [20] by increasing the amount of waveguide positional disorder. The excellent agreement between experimental observations and theoretical calculations is a testament to the accuracy of the waveguide fabrication technique.

\section{Random walk in waveguide arrays}

The propagation of an optical field along a lossless waveguide array with nearest-neighbor evanescent coupling can be described, in general, by the equation

$$
i \frac{d E_{n}}{d z}+\beta_{n} E_{n}+\mathscr{C}_{n, n+1} E_{n+1}+\mathscr{C}_{n, n-1} E_{n-1}=0,
$$

where $E_{n}$ is the electric field amplitude at the $n^{\text {th }}$ waveguide $(n=1,2, \cdots, N), \beta_{n}$ is the propagation constant of the $n^{\text {th }}$ waveguide, and $\mathscr{C}_{n, n \pm 1}$ is the coupling coefficient between adjacent waveguide elements. We assume lossless propagation and set $\mathscr{C}_{n, n \pm 1}=\mathscr{C}_{n \pm 1, n}$. The magnitude of the coupling coefficients depends exponentially on the separation between adjacent waveguides [19].

We begin by considering a periodic array $\left(\mathscr{C}_{n, n \pm 1}=\mathscr{C}_{o}\right)$ of identical $\left(\beta_{n}=\beta_{o}\right)$ waveguides, whereupon Eq. (1) simplifies to

$$
i \frac{d E_{n}}{d z}+\mathscr{C}_{o}\left(E_{n+1}+E_{n-1}\right)=0 .
$$

For single-input-site excitation $E_{n_{o}}=A_{0} \delta_{n, n_{o}}$ at $z=0$, the field in the $n^{\text {th }}$ waveguide is given by

$$
E_{n, n_{o}}(z)=A_{0} i^{n-n_{o}} J_{n-n_{o}}\left(2 \mathscr{C}_{o} z\right)
$$

where $J_{n}(x)$ represents a Bessel function of order $n$, and the output intensity distribution is

$$
I_{n, n_{o}}(z) \propto\left|J_{n-n_{o}}\left(2 \mathscr{C}_{o} z\right)\right|^{2} .
$$

This output distribution exhibits two off-center lobes where most of the optical energy is concentrated, and whose distance from the transverse location of the excitation site increases linearly with the propagation length along the array (see Fig. 1a). This is characteristic of discrete diffraction [5], which is in stark contrast to free-space diffraction where most of the light is concentrated in a central lobe.

\#145189 - \$15.00 USD Received 1 Apr 2011; revised 15 May 2011; accepted 26 May 2011; published 29 Jun 2011

(C) 2011 OSA

4 July 2011 / Vol. 19, No. 14 / OPTICS EXPRESS 13638 
Disorder can be introduced into a waveguide array by one of two strategies. In the first, one randomly changes the waveguide width, while keeping the distance between waveguide centers constant. As a result, the propagation constants $\beta_{n}$ vary from one waveguide to another in the range $\beta_{o} \pm \Delta$, while the coupling coefficients are approximately constant, $\mathscr{C}_{n, n \pm 1}=\mathscr{C}_{0} \quad[13$, 14]. This disordered array corresponds to the diagonal-disorder model in Anderson's original formulation. In the second strategy, the waveguides are all identical, but the separation between adjacent waveguides is randomized. The propagation constant is the same for all the waveguides $\beta_{n}=\beta_{o}$, while the coupling coefficients become random in the range $\mathscr{C}_{o} \pm \Delta$. Such an array corresponds to the off-diagonal-disorder model. In this paper, we focus on waveguide arrays involving off-diagonal disorder. Propagation in such an array is described by the following
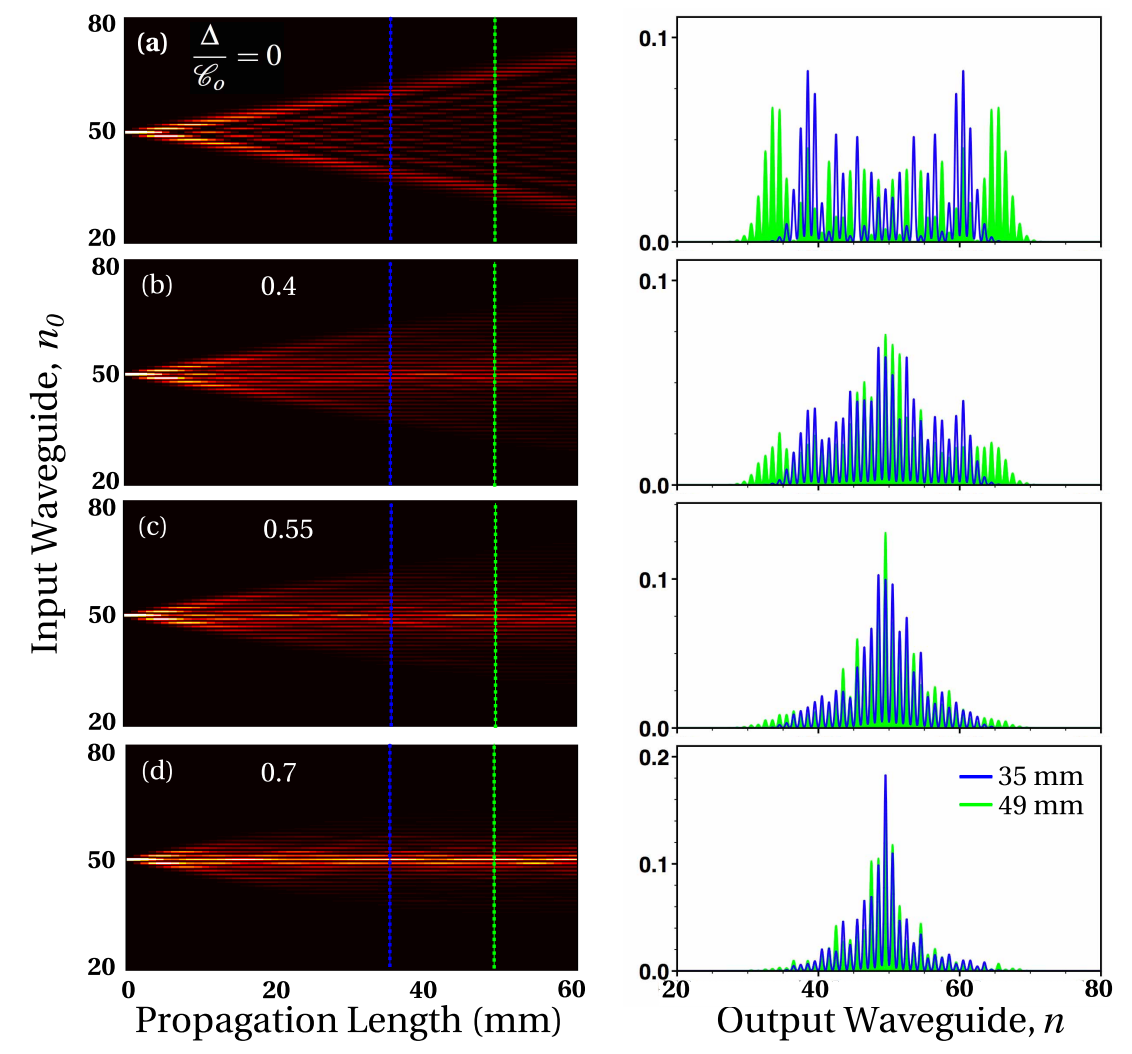

Fig. 1. Numerical simulation of optical field propagation when light is injected into the $51^{\text {st }}$ waveguide in a 101-waveguide array. The arrays used in (a) to (d) have an increasing degree of disorder. Each plot results from averaging the intensities of 41 realizations of random disordered arrays described by a uniform probability distribution function having a mean value $\mathscr{C}_{0}=1.8 \mathrm{~cm}^{-1}$ and width $2 \Delta$, for disorder parameters $\Delta / \mathscr{C}_{0}=0,0.4,0.55$, and 0.70 , respectively. The average output intensity distributions for propagation lengths $35 \mathrm{~mm}$ (blue) and $49 \mathrm{~mm}$ (green), respectively, corresponding to the lengths of the two samples used, are shown on the right.

equation,

$$
i \frac{d E_{n}}{d z}+\mathscr{C}_{n, n+1} E_{n+1}+\mathscr{C}_{n, n-1} E_{n-1}=0
$$



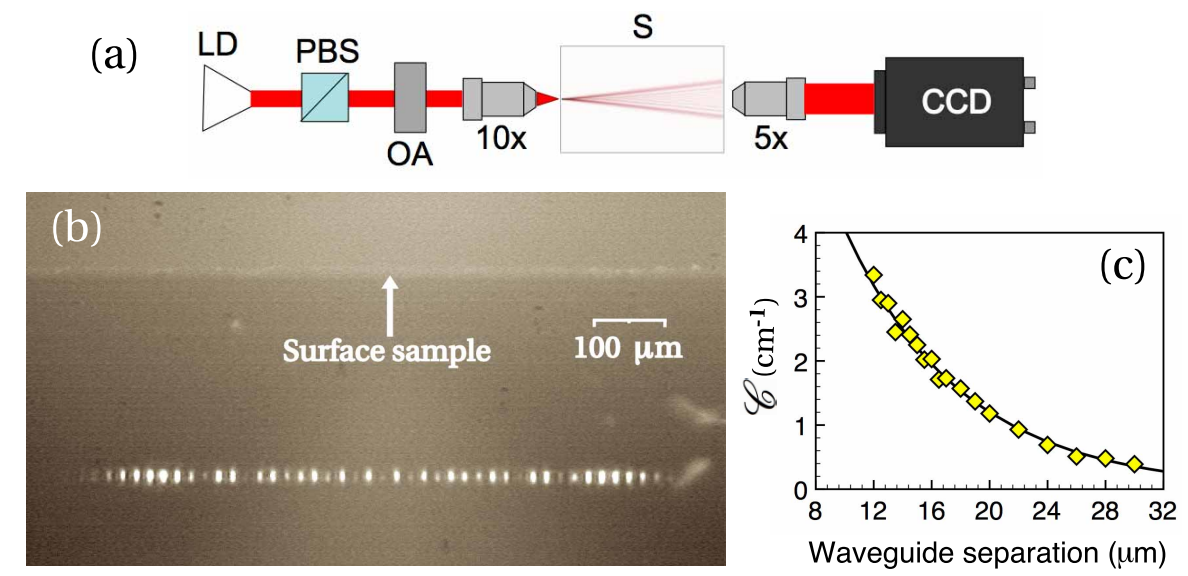

Fig. 2. (a) Experimental setup. LD: laser diode (780 nm); PBS: polarizing beam splitter; OA: optical attenuator; $5 \times(\mathrm{NA}=0.1)$ and $10 \times(\mathrm{NA}=0.25)$ microscope objectives; $\mathrm{S}$ : waveguide array sample. (b) CCD image of the periodic waveguide array output (period $=17 \mu \mathrm{m}$, and distance from the top of fused silica slab $\approx 250 \mu \mathrm{m}$ ) when a single waveguide is excited at the input. (c) Measured dependence of the coupling coefficient $\mathscr{C}$ on the waveguide separation for directional couplers fabricated with the same parameters as the arrays.

In Figs. 1b-d we present numerical simulations of the intensity of a field propagating along such an array averaged over 41 realizations of the random disordered parameters $\mathscr{C}_{n, n \pm 1}$, chosen according to a uniform probability distribution with a mean value $\mathscr{C}_{O}$ and width $2 \Delta$, for disorder parameters $\Delta / \mathscr{C}_{0}=0,0.4,0.55$, and 0.70 . Note the transition from extended (Fig. 1a) to exponentially localized (Fig. 1d) optical states with increasing disorder.

\section{The waveguide array}

The waveguides used in this study were fabricated using 800-nm-wavelength femtosecondlaser pulses focused at a depth of $\approx 250$ microns below the surface of polished bulk fused-silica glass $[18,19]$, inducing permanent refractive index changes.

A computer-controlled positioning system allows one to write waveguides [18] of transverse size $4 \times 12 \mu \mathrm{m}$. At a wavelength of $800 \mathrm{~nm}$, these are single-mode waveguides with $\mathrm{NA}=$ 0.06 [19]. We prepared two identical samples each consisting of four waveguide arrays, but having different lengths, $35 \mathrm{~mm}$ and $49 \mathrm{~mm}$, referred to hereon as short and long samples, respectively. The waveguides in all of the arrays in both samples are identical. Each array consists of 101 waveguides with nearest-neighbor evanescent coupling. The first array in each sample is periodic with inter-waveguide separation of $17 \mu \mathrm{m}$ (numerical simulation of optical field propagation when light is injected into a single waveguide of the periodic array is shown in Fig. 1a), corresponding to a coupling coefficient $\mathscr{C}_{O} \simeq 1.8 \mathrm{~cm}^{-1}$ (Fig. 2c). The other arrays are disordered with random (off-diagonal) coupling coefficients. The values of the coupling coefficients in each array are described by uniform probability distribution functions all having the same mean value $\mathscr{C}_{o}$, but with increasingly larger width $2 \Delta$ (Fig. 1b-d). According to the exponential dependence of $\mathscr{C}_{0}$ on the waveguide separation (Fig. 2c), such a uniform distribution of coupling coefficients can be generated by imposing an exponential distribution on the separation [19]. 


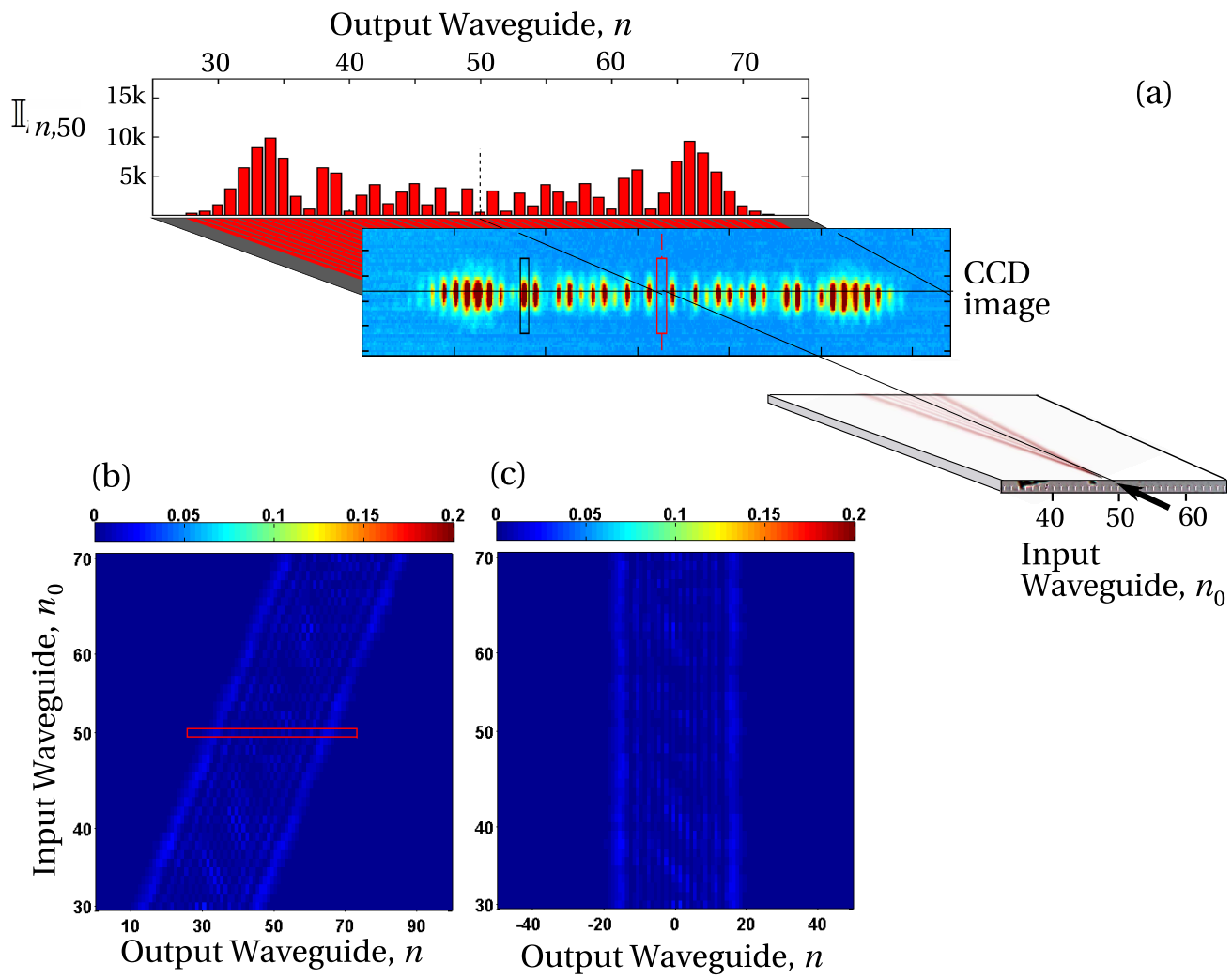

Fig. 3. Data acquisition and analysis. Panel (a) presents data for light injected into the $50^{\text {th }}$ waveguide $\left(n_{o}=50\right)$ of the long periodic array. The intensity at the output of the waveguide array is captured with a CCD camera (shown in the middle). The image is then post-processed to extract the discrete intensity distribution $\left(\mathbb{I}_{n, 50}\right)$ by integrating over rectangular areas $10 \times 30$ pixel each centered on the center of each waveguide $(n)$ shown as the black rectangle. The central red rectangle on the CCD image in panel (a) indicates the location of the excitation site. The discrete intensity distribution, $\mathbb{I}_{n, 50}$ is shown as the red bar-plot. The brightness image in panel (b) displays the distribution, $\mathscr{I}_{n, n_{o}}$ of the intensity of the light measured at the output of the waveguides $(n)$ when only waveguide $n_{o}$ is illuminated. The red rectangle on panel (b) indicates the output distribution for light injected into the $50^{\text {th }}$ waveguide. In panel (c) the displaced distribution, $\mathscr{I}_{n+n_{o}, n_{o}}$ is shown. Each distribution of the measured intensity is displaced such that it is centered about the illuminated waveguide. Only the middle 41 waveguides are illuminated (one at a time) with the ordinate marking the illuminated waveguide. 


\section{Optical measurement system}

The experimental setup used to observe the transition to Anderson localization in the abovedescribed optical waveguide arrays is shown in Fig. 2. A horizontally polarized beam from a diode laser at $780 \mathrm{~nm}$ is attenuated and focused by a $10 \times$ microscope objective $(\mathrm{NA}=0.25)$ into a single waveguide in the array (see Fig. 2a). The waveguide array output is imaged on a CCD camera using a $5 \times$ microscope objective $(\mathrm{NA}=0.1)$. A typical output intensity distribution for single-site excitation in the periodic array in the long sample is shown in Fig. 2b, demonstrating clearly the expected discrete diffraction pattern.

The data recording and analysis procedure is sketched in Fig. 3. A single waveguide is illuminated and the intensity of the light at the output of the waveguide array is captured by the CCD camera. The realization shown in Fig. 3a was obtained by injecting light into the $50^{\text {th }}$ waveguide in the long-sample periodic array. The $2 \mathrm{D}$ image was post-processed to extract a discretized 1D intensity distribution. A rectangle of size $10 \times 30$ pixels that covers the image of a waveguide was integrated and a background term was subtracted. The resulting discrete intensity distribution $\mathbb{I}_{n, 50}$ is shown as the red-bar plot.

(a)

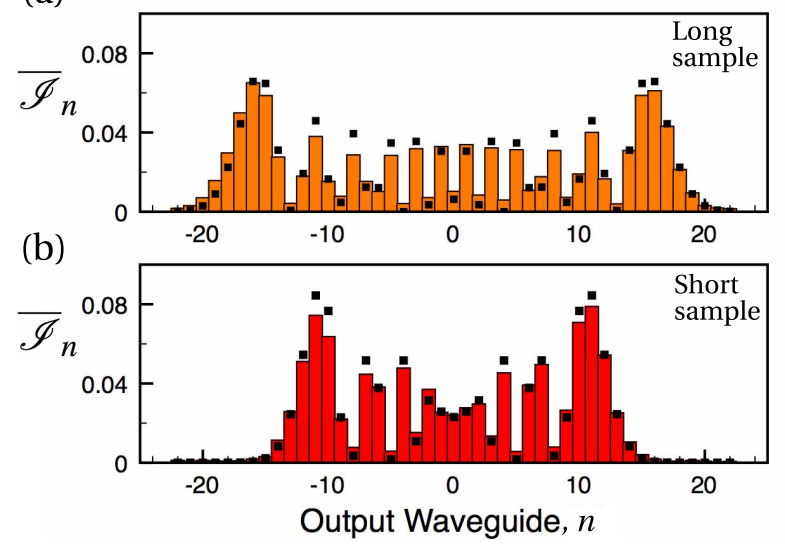

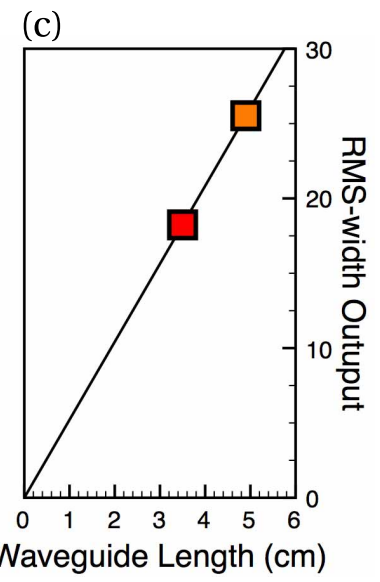

Fig. 4. Average displaced distribution $\overline{\mathscr{I}}_{n}$ for long (a) and short (b) periodic waveguide arrays. The black squares represent a theoretical best-fit with $\mathscr{C}_{0} \approx 1.79 \mathrm{~cm}^{-1}$ and $1.80 \mathrm{~cm}^{-1}$ for the short and long arrays, respectively. The root-mean-square (RMS) width of the experimental distributions $(\approx 18.0$ and $\approx 25.4$ ) are shown in $(\mathrm{c})$ as function of the array length. The line represents the best-fit to the linear ballistic expansion as a function of the array length with $\mathscr{C}_{o} \approx 1.81 \mathrm{~cm}^{-1}$.

The uniformity of the waveguide losses is attested by the fact that the total output power $\mathbb{P}_{n_{o}}=\sum_{n} \mathbb{I}_{n, n_{o}}=\mathbb{P}$ is constant $\forall n_{o}$ (for fixed input power). We normalized the output intensity with respect to $\mathbb{P}, \mathscr{I}_{n, n_{o}}=\mathbb{I}_{n, n_{o}} / \mathbb{P}$ and then averaged the output distributions for different excitation sites after shifting them by the index of that excitation site (see Fig. 3c),

$$
\overline{\mathscr{I}}_{n}=\sum_{n_{0}} \mathscr{I}_{n+n_{0}, n_{0}} .
$$

The resulting averages are shown in Fig. 4 for the short and long periodic arrays. This procedure is justified since the characteristics of the array are shift invariant. This necessitates excluding edge effects which occur if the ends of the array are excited, as investigated by Szameit et al. [15]. Therefore, we excite the input waveguides $n_{o}=31 \ldots 71$, which guarantee that the output intensity distribution does not extend to the edges of the arrays. A best-fit for these 

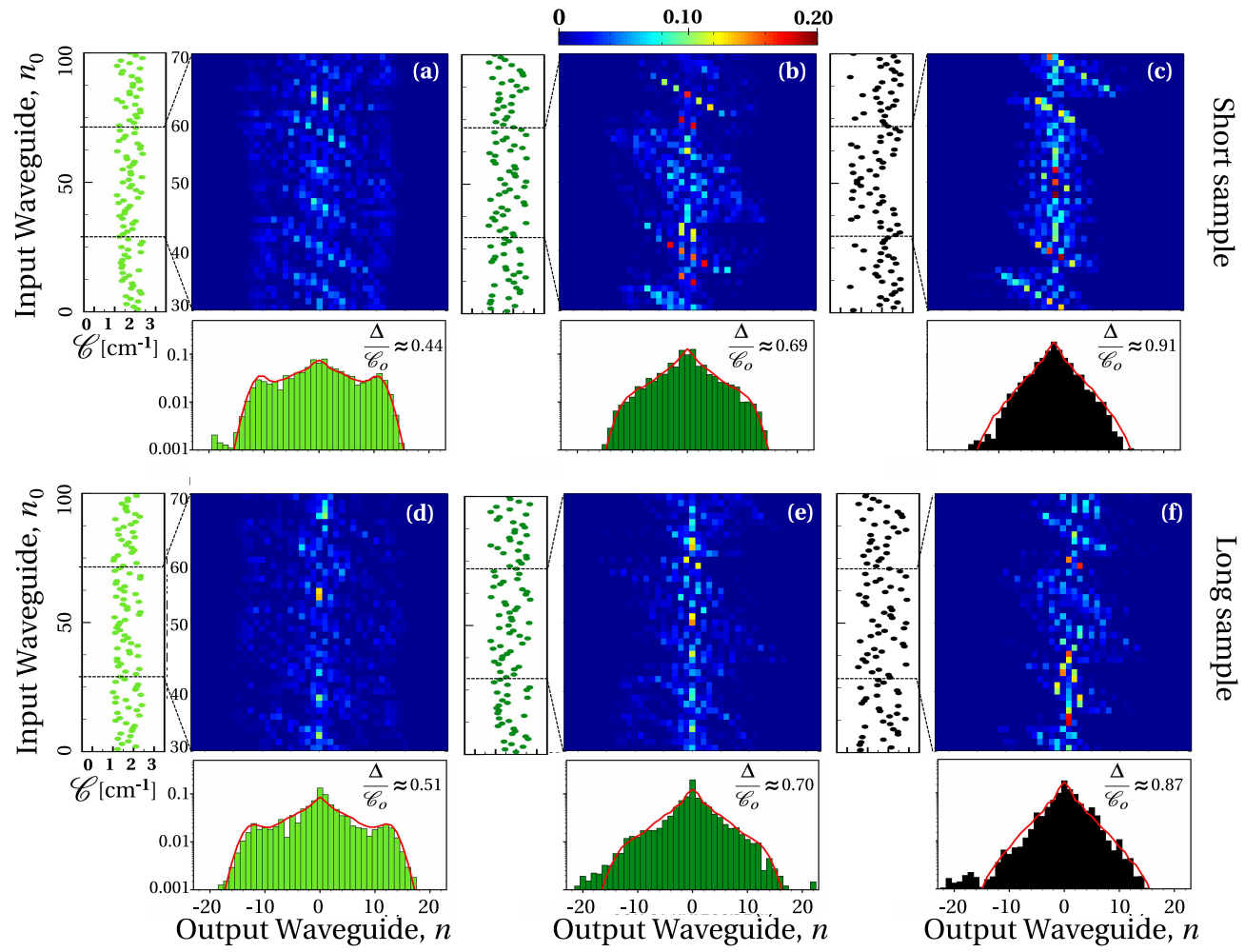

Fig. 5. Effect of disorder on the propagation of light through 101-waveguide arrays in the short (first row, a-c) and long (second row, d-f) samples. The two rows correspond to disorder parameters $\Delta / \mathscr{C}_{0} \approx 0.44,0.69$ and 0.91 for the short sample, and $0.51,0.70$ and 0.87 for the long sample. The color plot in each panel shows the displaced distributions $\mathscr{I}_{n+n_{o}, n_{o}}$ at the output. Each row in the plot corresponds to the output intensity distribution for a single point excitation at $n_{o}$ after shifting it by $n_{o}$. Only the middle 41 waveguides are illuminated (one at a time) with the ordinate marking the illuminated waveguide. The average of the displaced distributions, $\overline{\mathscr{I}}_{n}$, for all 41 waveguides is plotted at the bottom of each panel with the red line showing the result of a numerical simulation with $\mathscr{C}_{0}$ and $\Delta$ as fitting parameters.

distributions to the theoretical expectation $\left|J_{n}\left(2 \mathscr{C}_{0} z\right)\right|^{2}$ allows us to evaluate the coupling coefficient to be $\mathscr{C}_{0} \approx 1.79 \mathrm{~cm}^{-1}$ and $1.80 \mathrm{~cm}^{-1}$ for the short and long arrays, respectively, defined by numerical simulation fittings. Further confirmation of our results comes from verifying that the separation of the lobes in the ballistic expansion increases linearly with sample length. We have evaluated the root-mean-square (RMS) width of the experimental distributions $\mathscr{\mathscr { I }}_{n}$, and fitted them with the coupling coefficient $\mathscr{C}_{0}$ as the only free parameter, as shown in Fig. 4.

\section{Anderson localization in waveguide arrays with off-diagonal disorder}

We next proceed to examine wave propagation through waveguide arrays with off-diagonal disorder. The coupling coefficients between adjacent waveguides in a single array were chosen such that they belong to a uniform probability distribution function having mean value $\mathscr{C}_{0}$. The width of the distribution $2 \Delta$ increases from one array to the next, corresponding to increasing disorder. The values of $\mathscr{C}_{0}$ and $\Delta$ have been determined by fitting the experimental data with 
numerical simulations: $\mathscr{C}_{0}\left(\approx 1.79 \mathrm{~cm}^{-1}\right.$ and $1.50 \mathrm{~cm}^{-1}$ for the short and long arrays, respectively) defines the distance between the lobes of the ballistic expansion, which is still visible in Fig. 5a and 5d, while $\Delta$ defines the central exponential peak. The disorder parameters for our arrays are found to be $\Delta / \mathscr{C}_{0} \approx 0.44,0.69$ and 0.91 for the short sample, and $0.51,0.70$ and 0.87 for the long sample. The experimental setup and data analysis procedure used with the disordered arrays were identical to those described above for the periodic arrays (see Fig. 2) after accounting for the random locations of the waveguides in these off-diagonal disordered arrays. We have also used here the same post-processing data analysis to obtain the displaced distributions $\mathscr{I}_{n+n_{o}, n_{o}}$ and the average displaced distribution $\overline{\mathscr{I}}_{n}$ for each array.

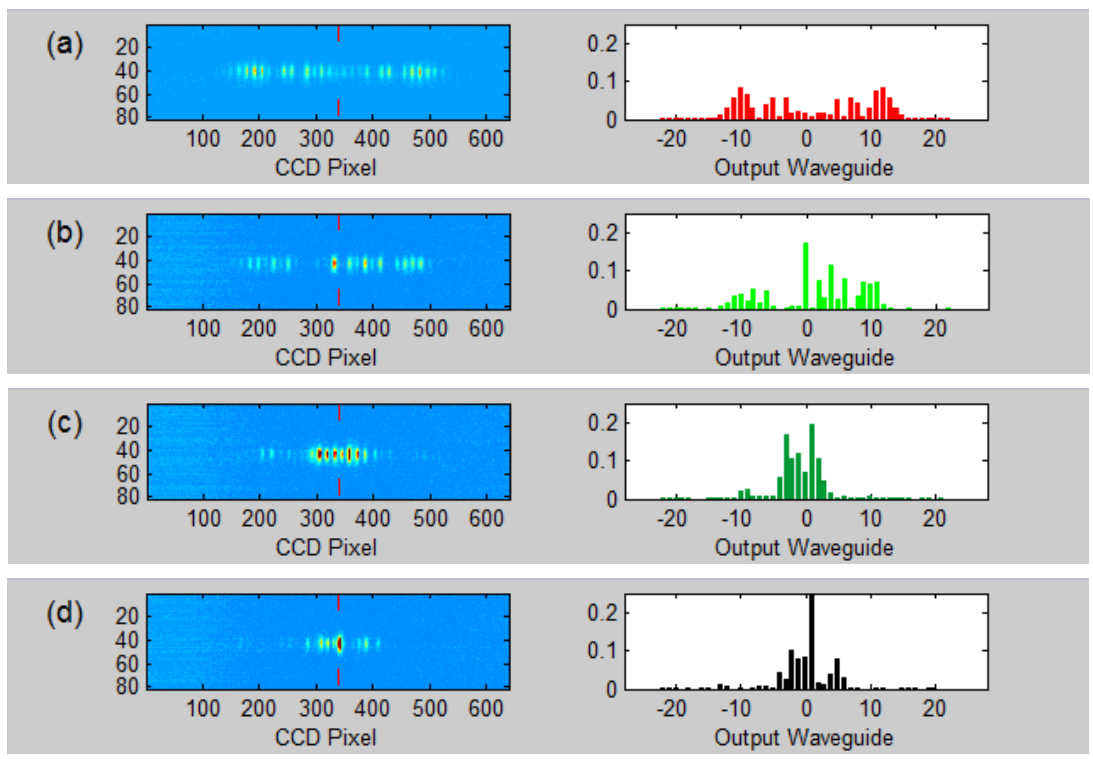

Fig. 6. Single-frame excerpts from video recordings. On the left we display the recorded intensity distribution at the output of the short waveguide array when the middle 41 input waveguides are illuminated one at a time, while on the right the cumulative averaged discrete intensity distribution is updated. (a) Periodic array (Media 1); (b) array with disorder parameter $\Delta / \mathscr{C}_{0} \approx 0.44$ (Media 2); (c) array with $\Delta / \mathscr{C}_{0} \approx 0.69$ (Media 3); (c) array with $\Delta / \mathscr{C}_{o} \approx 0.91$ (Media 4).

As we repeat the experiment in arrays with progressively larger off-diagonal coupling disorder, shortening the localization length with respect to the ballistic spreading, we observe at the array outputs a clear enhancement of the exponentially localized (Anderson-localized) optical states for both samples [20]. As shown in Fig. 5, we observe that the ballistic expansion in the periodic array evolves, with increasing disorder, into an intermediate regime at $\Delta / \mathscr{C}_{0} \sim 0.5$ that exhibits characteristics of both extended and localized states (Fig. 5a,d). Finally Anderson localization is clearly evident at $\Delta / \mathscr{C}_{0} \sim 0.9$ (Fig. 5c,f).

It is worth noting that as we scan the beam injected into individual waveguides across a disordered array, besides the shift due to the scanned input, the output intensity distribution changes. This results from the fact that the spreading optical field encounters a random coupling environment as we move across the array. In contrast, the individual realizations at the output of the periodic array are almost all identical (modulo the shift). Anderson localization is then established for the disordered array by averaging the different realizations resulting from spatial 
scanning the input beam. These features are brought together in the movies in Fig. 6. On the left we depict the individual output intensity distributions resulting from scanning the excited waveguides at the input, and in the right we display an updated cumulative average. In the case of the periodic array, averaging has little effect.

The localized states observed by averaging over multiple realizations of the gradually increased disorder is demonstrated in Fig. 7. The RMS-widths of the output intensity distribution measured for the two arrays with different length are compared to numerical simulations for two methods of statistical averaging over the waveguide disorder. We note that our results demonstrate that ensemble statistical averaging, achieved by coupling into a single waveguide in a set of independent disordered arrays (sampling average) is equivalent to spatial scanning through multiple waveguides in the same off-diagonally disordered array (shifted average). Finally to highlight the exponential decay of the Anderson-localized state away from its center, we plot in log-scale the average displaced distribution $\overline{\mathscr{I}}_{n}$ for short and long samples in the inset of Fig. 7. The exponential decay fits until we reach the noise level of the data.

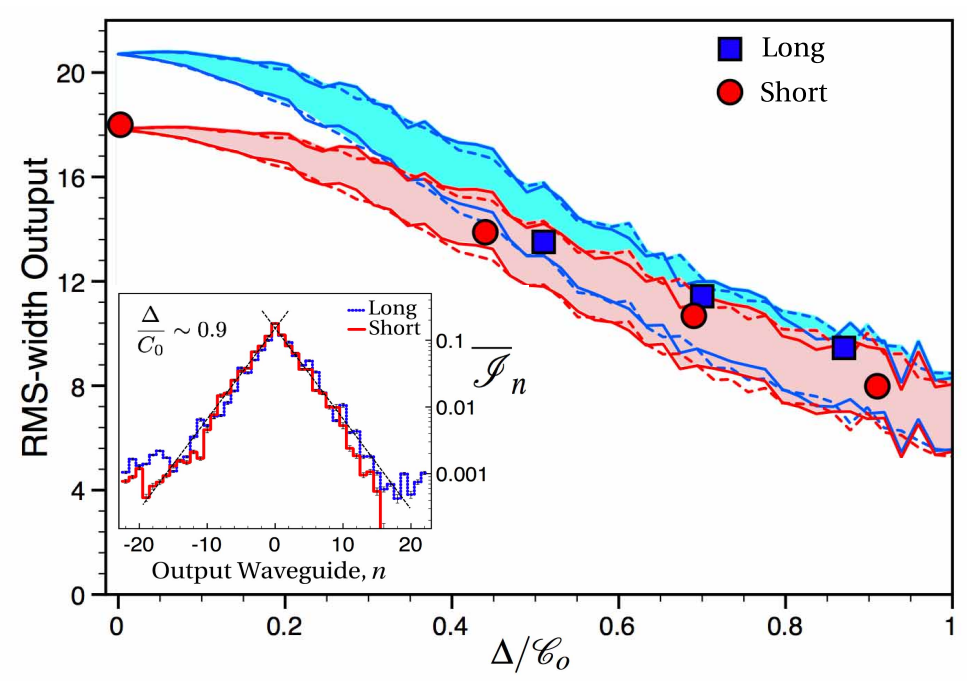

Fig. 7. RMS width as function of the disorder parameter $\Delta / \mathscr{C}_{0}$ for the short (red-circle symbols) and long array (blue-square symbols). The colored bands represent the range of values of the RMS-width a standard deviation around the mean value. For each value of the disorder parameter, RMS-width and its standard deviation have been evaluated by averaging over $21 \times 40$ disorder realizations for the sampling average approach (dashed lines), while 21 disorder realizations and 40 shifted input waveguides have been considered for the shifting average approach (solid lines). Inset: average displaced distribution, $\overline{\mathscr{I}}_{n}$ for short and long arrays with disorder parameter $\Delta / \mathscr{C}_{O} \sim 0.9$. The log-scale plot highlights the exponential decay of the Anderson-localized states. The dotted-lines are a guide for the eye.

\section{Conclusion}

We have observed the gradual passage from extended to Anderson-localized states in nearinfrared light propagation through waveguide arrays of different lengths having a uniformly distributed off-diagonal coupling disorder. Precise fabrication techniques have allowed us to control the disorder parameter and enabled us to obtain experimental measurements confirming theoretical predictions with good accuracy, including the exponential behavior of the Andersonlocalized state. We have supported the experimental results with numerical simulations for both 
shifting and sampling statistical averaging methods and shown that both methods yield equivalent results of the same precision.

\section{Acknowledgments}

This work was supported by CREOL, The College of Optics and Photonics, at the University of Central Florida, and Deutsche Forschungsgemeinschaft (Research Unit 532 and Leibniz program).

\#145189 - \$15.00 USD Received 1 Apr 2011; revised 15 May 2011; accepted 26 May 2011; published 29 Jun 2011 (C) 2011 OSA 